\title{
Cyclic Labellings with Constraints at Two Distances
}

\author{
R A Leese \\ Smith Institute for Industrial Mathematics and System Engineering \\ PO Box 183 \\ Guildford GU2 7GG \\ leese@maths.ox.ac.uk \\ and \\ S D Noble \\ Department of Mathematical Sciences \\ Brunel University \\ Uxbridge UB8 3PH \\ mastsdn@brunel.ac.uk
}

Submitted: Jan 23, 2002; Accepted: Sep 30, 2003; Published: Feb 14, 2004.

MR Subject Classifications: 05C78, 05C15

\begin{abstract}
Motivated by problems in radio channel assignment, we consider the vertexlabelling of graphs with nonnegative integers. The objective is to minimize the span of the labelling, subject to constraints imposed at graph distances one and two. We show that the minimum span is (up to rounding) a piecewise linear function of the constraints, and give a complete specification, together with the associated optimal assignments, for trees and cycles.
\end{abstract}




\section{Introduction}

An important task in the management of the radio spectrum is the assignment of radio channels to transmitters in ways that avoid unacceptable interference. In broad terms, one must avoid assigning channels with close frequencies to transmitters with close locations, an observation that leads to the following mathematical model.

Take a graph $G$, in which each vertex represents a transmitter and the edges represent geographic proximity. A channel assignment is a map $f: V(G) \rightarrow\{0,1,2, \ldots, s-1\}$ for some integer $s$, which is called the span of $f$. It is natural that the range of $f$ should be restricted to nonnegative integers, since in practice radio channels are made available at equally spaced frequencies. The span captures the amount of spectrum used. To describe the constraints imposed on $f$ to avoid interference, we measure the geographic separation of transmitters $u$ and $v$ by the graph distance $d_{G}(u, v)$ in $G$. This paper will not consider constraints acting beyond a graph distance of two. Suppose that $J(G)=\{(u, v) \mid u, v \in$ $\left.V(G), d_{G}(u, v)=1\right\}$ and $K(G)=\left\{(u, v) \mid u, v \in V(G), d_{G}(u, v)=2\right\}$ are the sets of nearest neighbours and next-to-nearest neighbours, respectively. Then the constraints take the form

$$
|f(u)-f(v)|_{s} \geq j \quad \forall(u, v) \in J(G)
$$

and

$$
|f(u)-f(v)|_{s} \geq k \quad \forall(u, v) \in K(G),
$$

where, on the left hand side, we have used the cyclic channel metric defined by

$$
|f(u)-f(v)|_{s}=\min \{|f(u)-f(v)|, s-|f(u)-f(v)|\} .
$$

In keeping with the idea of a trade-off between spectral and spatial separations, we assume that $k$, the minimum spectral separation at spatial distance 2 , is no larger than $j$, the minimum separation at distance 1 .

The adoption of the cyclic channel metric, instead of a straightforward absolute difference, has both practical and theoretical motivation. First, it allows easy extension of the assignment to one that gives $m$ channels to each transmitter $v$, namely the channels $\{f(v)+i s: 0 \leq i<m\}$; multiple coverage of this type is often needed in practice. Secondly, it means that no channel has special status by virtue of having neighbours in the spectrum on only one side; experience suggests that explicit results can then be obtained in a wider range of situations.

We shall concentrate on determining, for given $G$, the minimum span of all assignments $f$ satisfying constraints (1) and (2). The minimum span will be denoted $\sigma(G ; j, k)$, emphasizing the dependence on $j$ and $k$.

It is often helpful to consider the following 'relaxed' version of the problem. Suppose that the channels may take any nonnegative real values, denoted $\hat{f}(v)$, in place of the integer-valued $f(v)$ and similarly that $j$ and $k$ may take nonnegative real values. We now think of the assignment as a map $\hat{f}: V(G) \rightarrow[0, \hat{s})$, where $\hat{s}$, the span of $\hat{f}$, may also be nonintegral. Equations (1)-(3) still hold, provided $f$ and $s$ are replaced by $\hat{f}$ and $\hat{s}$, respectively. The minimum span for this problem will be denoted $\hat{\sigma}(G ; j, k)$. 
Proposition 1 For given $G, \hat{\sigma}(G ; j, k)$ is a continuous, piecewise linear function of $j$ and $k$, with non-negative coefficients.

Proof First, suppose that an ordering is imposed on the image of $\hat{f}$, using a permutation $\pi$, thought of as a bijection from $\{1,2, \ldots,|V(G)|\}$ to $V(G)$. Explicitly, we require $\hat{f}_{1} \leq$ $\hat{f}_{2} \leq \cdots \leq \hat{f}_{|V(G)|}$, where $\hat{f}_{i}$ is shorthand for $\hat{f}(\pi(i))$. The minimum span for the problem with ordering $\pi$, denoted $\hat{\sigma}(G, \pi ; j, k)$, is the value of the following linear program, in which the variables are $\left\{\hat{f}_{i}: 1 \leq i \leq|V(G)|\right\}$ and $z$ :

$$
\begin{array}{rll}
\text { minimize } & z & \\
\text { subject to } & \hat{f}_{q}-\hat{f}_{p} \geq j & \text { for } q>p \text { and } d_{G}(\pi(p), \pi(q))=1 \\
& \hat{f}_{p}-\hat{f}_{q}+z \geq j & \text { for } q>p \text { and } d_{G}(\pi(p), \pi(q))=1 \\
& \hat{f}_{q}-\hat{f}_{p} \geq k & \text { for } q>p \text { and } d_{G}(\pi(p), \pi(q))=2 \\
& \hat{f}_{p}-\hat{f}_{q}+z \geq k & \text { for } q>p \text { and } d_{G}(\pi(p), \pi(q))=2 \\
& z, \hat{f}_{i} \geq 0 . &
\end{array}
$$

The standard theory of linear programming guarantees that $\hat{\sigma}(G, \pi ; j, k)$ is a continuous, piecewise linear function of $j$ and $k$ for fixed $G$ and $\pi$. It is now clear that the same statement is true of $\hat{\sigma}(G ; j, k)$, since $\hat{\sigma}(G ; j, k)=\min _{\pi} \hat{\sigma}(G, \pi ; j, k)$ and the envelope of a (finite) set of continuous, piecewise linear functions is again continuous and piecewise linear. For fixed $j, \hat{\sigma}$ is an increasing function of $k$ and similarly for fixed $k, \hat{\sigma}$ is an increasing function of $j$. Thus in each linear component of $\hat{\sigma}$, the coefficients of both $j$ and $k$ must be positive.

We now show that when $j$ and $k$ are both positive integers,

$$
\sigma(G ; j, k)=\lceil\hat{\sigma}(G ; j, k)\rceil .
$$

Suppose that $\{\hat{f}(v): v \in V(G)\}$ is an optimal assignment for the relaxed problem, i.e. one with span $\hat{\sigma}(G ; j, k)$. Define an integer-valued assignment $f$ by setting $f(v)=\lfloor\hat{f}(v)\rfloor$ for each $v \in V(G)$. Since $j$ and $k$ are integer-valued, $f$ satisfies (1) and (2) with span $\lceil\hat{\sigma}(G ; j, k)\rceil$. Explicitly,

$$
\begin{aligned}
& |\hat{f}(u)-\hat{f}(v)| \geq j \Rightarrow|f(u)-f(v)| \geq j \\
& |\hat{f}(u)-\hat{f}(v)| \geq k \Rightarrow|f(u)-f(v)| \geq k
\end{aligned}
$$

and

$$
\begin{aligned}
& \hat{\sigma}-|\hat{f}(u)-\hat{f}(v)| \geq j \quad \Rightarrow\lceil\hat{\sigma}\rceil-|f(u)-f(v)| \geq j \\
& \hat{\sigma}-|\hat{f}(u)-\hat{f}(v)| \geq k \Rightarrow\lceil\hat{\sigma}\rceil-|f(u)-f(v)| \geq k .
\end{aligned}
$$

Hence $\sigma(G ; j, k) \leq\lceil\hat{\sigma}(G ; j, k)\rceil$, but since $\sigma$ is integer-valued and bounded below by $\hat{\sigma}$ we in fact have

$$
\sigma(G ; j, k)=\lceil\hat{\sigma}(G ; j, k)\rceil,
$$

as required. 
We note here the following relation involving $\hat{\sigma}$ :

$$
\hat{\sigma}(G ; j, k)=j \hat{\sigma}\left(G ; 1, \frac{k}{j}\right) .
$$

The value $\hat{\sigma}(G ; 1,0)$ is a quantity usually called the circular chromatic number [6] and denoted $\chi_{c}(G)$. For the cases considered here, we have $\chi_{c}(T)=2, \chi_{c}\left(C_{2 r}\right)=2$ and $\chi_{c}\left(C_{2 r+1}\right)=2+(1 / r)$, for trees $T$, even cycles $C_{2 r}$ and odd cycles $C_{2 r+1}$.

This paper extends the work of [2] where $\sigma$ is calculated for the line, square lattice and triangular lattice.

The remainder of the paper is arranged as follows. Sections 2, 3 and 4 give complete descriptions of $\sigma(G ; j, k)$, for all values of $j$ and $k$, when $G$ is a tree, an even cycle and an odd cycle, respectively. Section 5 contains concluding remarks.

\section{Trees}

We begin by stating a well-known lower bound on the span first introduced in [4]. Since the proof is very short we include it for completeness. For vertices $u$ and $v$ we define $c_{u v}$ to be the required separation of the labels they receive, that is

$$
c_{u v}=\left\{\begin{array}{cc}
j & \text { if } u \text { and } v \text { are adjacent } \\
k & \text { if } u \text { and } v \text { are distance two apart } \\
0 & \text { otherwise }
\end{array}\right.
$$

The lower bound is known as the Travelling Salesman Lower Bound since it involves finding the shortest Travelling Salesman Tour around a graph with edge weights $c_{u v}$.

Proposition 2 Let $G$ be a graph with vertex set $\left\{v_{1}, v_{2}, \ldots, v_{n}\right\}$. Let $H$ be a subgraph of $G$ with $n^{\prime}$ vertices and let $\pi$ be an ordering of these vertices.

$$
\sigma(G ; j, k) \geq \min _{\pi} \sum_{i=1}^{n^{\prime}} c_{v_{\pi(i)}} v_{\pi(i+1)},
$$

where $\pi\left(n^{\prime}+1\right)=\pi(1)$.

Proof Since for any subgraph $H$ of $G, \sigma(H ; j, k) \leq \sigma(G ; j, k)$, it is enough to consider the case where $H=G$. Now

$$
\sigma(G, \pi ; j, k) \geq \sum_{i=1}^{n} c_{v_{\pi(i)} v_{\pi(i+1)}}
$$

and so

$$
\sigma(G ; j, k)=\min _{\pi} \sigma(G, \pi ; j, k) \geq \min _{\pi} \sum_{i=1}^{n} c_{v_{\pi(i)} v_{\pi(i+1)}} .
$$


This proposition allows us a short proof of the following theorem.

Theorem 1 Let $T$ be a tree with maximum degree $\Delta \geq 1$, then

$$
\sigma(T ; j, k)=2 j+(\Delta-1) k .
$$

Proof We first use Proposition 2 to show that $2 j+(\Delta-1) k$ is a lower bound for $\sigma$. Let $H$ be a subgraph of $T$ consisting of a vertex $r$ with degree $\Delta$ and all of its neighbours. In $H$ if $v \neq r$ then $c_{r v}=j$ and if $u, v \neq r$ then $c_{u v}=k$. Therefore the lower bound from Proposition 2 is $2 j+(\Delta-1) k$.

What remains is to construct a labelling with span $2 j+(\Delta-1) k$. We may assume that all vertices of $T$ except the leaves have degree $\Delta$. We begin by choosing a vertex $r$ of degree $\Delta$ and assigning it label 0 . The neighbours of $r$ are then labelled $j, j+$ $k, j+2 k, \ldots, j+(\Delta-1) k$. We now repeatedly choose a vertex $v$ of degree $\Delta$ which has itself already been labelled, but of its neighbours, only one vertex $u$ has been labelled. Without loss of generality we may assume that $u$ is labelled 0 and by the method used to construct the labelling we may assume $v$ is labelled $j+\alpha k$ for some integer $0 \leq \alpha \leq$ $\Delta-2$. There are still $\Delta-1$ neighbours of $v$ to label and the $\Delta-1$ labels in the set $\{k, 2 k, \ldots, \alpha k, 2 j+\alpha k, \ldots, 2 j+(\Delta-2) k\}$ are available for them.

\section{Even cycles}

In this section we focus on the case when $G$ is the even cycle $C_{2 r}(r \geq 2)$. We will however prove many of the results for cycles of any length so that we can make use of them in the next section. The vertex set of $C_{n}$ is denoted by $\left\{v_{0}, v_{1}, \ldots, v_{n-1}\right\}$. There is an edge between $v_{i-1}$ and $v_{i}$ for all $i$ with $1 \leq i \leq n-1$ and between $v_{n-1}$ and $v_{0}$. We begin by proving bounds on $\sigma\left(C_{2 r} ; j, k\right)$ and then specify the span for every value of $j, k$ and $r$.

Proposition 3 For any even cycle we have

$$
2 j+k \leq \sigma\left(C_{2 r} ; j, k\right) \leq 2 j+2 k \text {. }
$$

Proof To prove that $2 j+k$ is a lower bound for $\sigma\left(C_{2 r} ; j, k\right)$ we use Proposition 2 with $H$ being the path on three vertices.

To prove that $2 j+2 k$ is an upper bound for $\sigma\left(C_{2 r} ; j, k\right)$ we must construct a labelling with span $2 j+2 k$. For $C_{4}$ we just cyclicly label the vertices $0, j, 2 j+k, j+k$. For $C_{6}$ we cyclicly label the vertices $0, j, 2 j+k, j+k, k, j+2 k$. For larger even cycles we either label with repeated copies of the $C_{4}$ labelling or with one copy of the $C_{6}$ labelling followed by repeated copies of the $C_{4}$ labelling. 
Figure 1: Graph of $\hat{\sigma} / j$ against $k / j$ for $C_{8}$

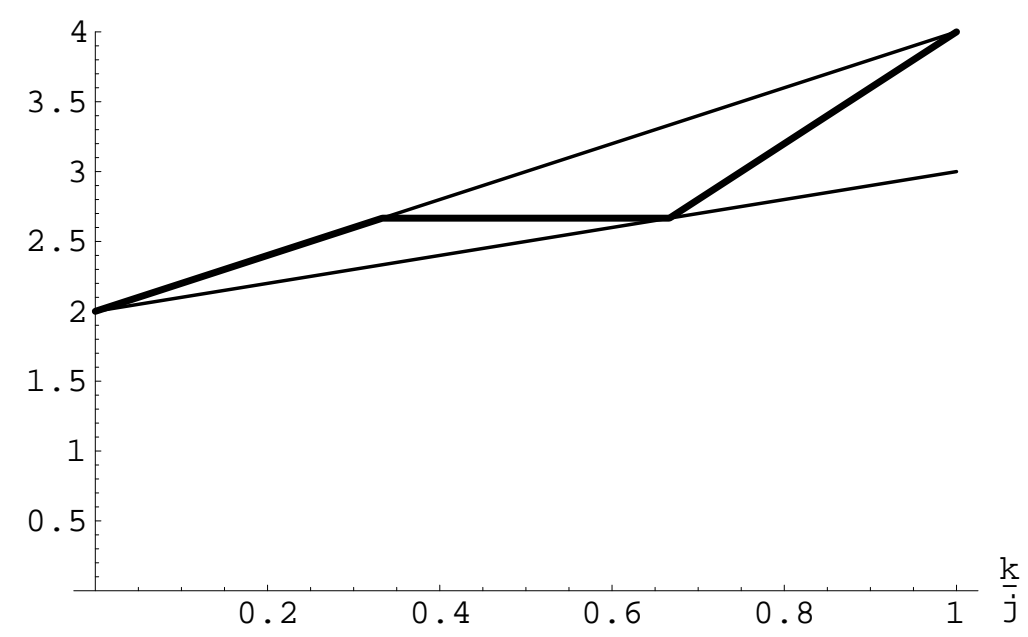

Theorem 2 Let $r, j$ and $k$ be positive integers with $j \geq k$, then

$$
\sigma\left(C_{2 r} ; j, k\right)= \begin{cases}2 j+2 k & 0 \leq \frac{k}{j} \leq \frac{1}{r-1} \\ \left\lceil\frac{2 r j}{r-1}\right\rceil & \frac{1}{r-1}<\frac{k}{j} \leq \frac{2}{r-1} \\ \left\lceil\frac{r k}{r-\alpha}\right\rceil & \frac{2(r-\alpha)}{\alpha}<\frac{k}{j} \leq \frac{2(r-\alpha)}{\alpha-1} \\ & \alpha=r-1, r-2, \ldots,\left\lceil\frac{1}{3}(2 r+1)\right\rceil \\ \left\lceil\frac{2 r j}{\alpha}\right\rceil & \frac{2(r-\alpha-1)}{\alpha}<\frac{k}{j} \leq \frac{2(r-\alpha)}{\alpha} \\ & \alpha=r-2, r-3, \ldots,\left\lceil\frac{1}{3}(2 r-1)\right\rceil .\end{cases}
$$

The graphs of $\hat{\sigma} / j$ as a function of $k / j$ are shown in Figures 1-3. The lines $y=2 x+1$ and $y=2 x+2$ corresponding to spans $2 j+k$ and $2 j+2 k$, respectively the lower and upper bounds from Proposition 3, are also shown.

We first describe two types of labellings which achieve the span in the theorem and then later show that they are optimal. Since these labellings will be equally important for odd cycles we describe them for a general length cycle.

We call the first type of labelling a one-step labelling. Let $\alpha$ be an integer such that

$$
1 \leq \alpha \leq \frac{n j}{2 j+k}
$$


Figure 2: Graph of $\hat{\sigma} / j$ against $k / j$ for $C_{10}$

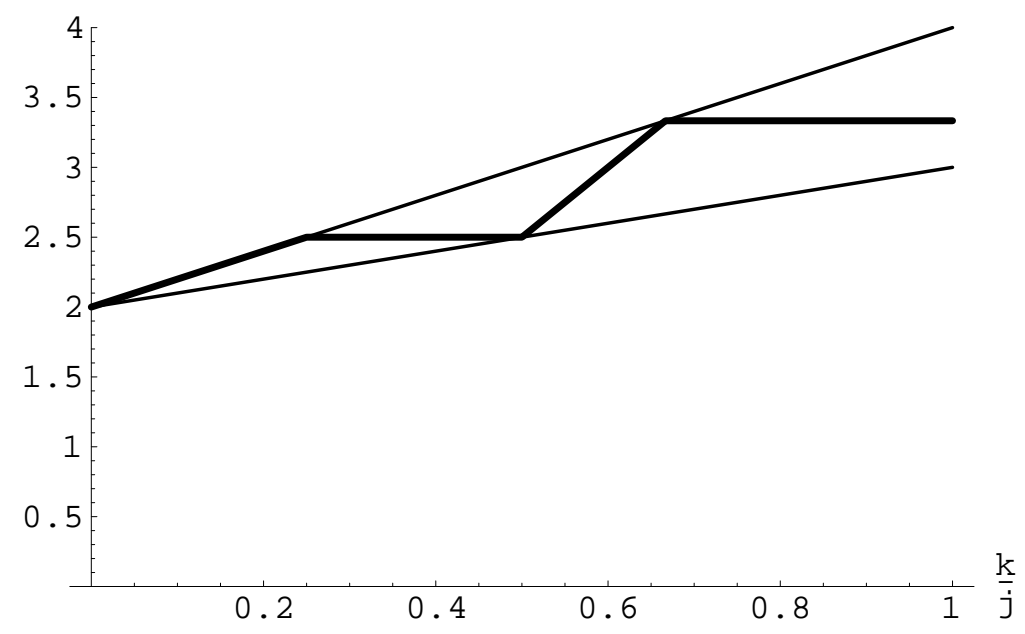

Figure 3: Graph of $\hat{\sigma} / j$ against $k / j$ for $C_{16}$

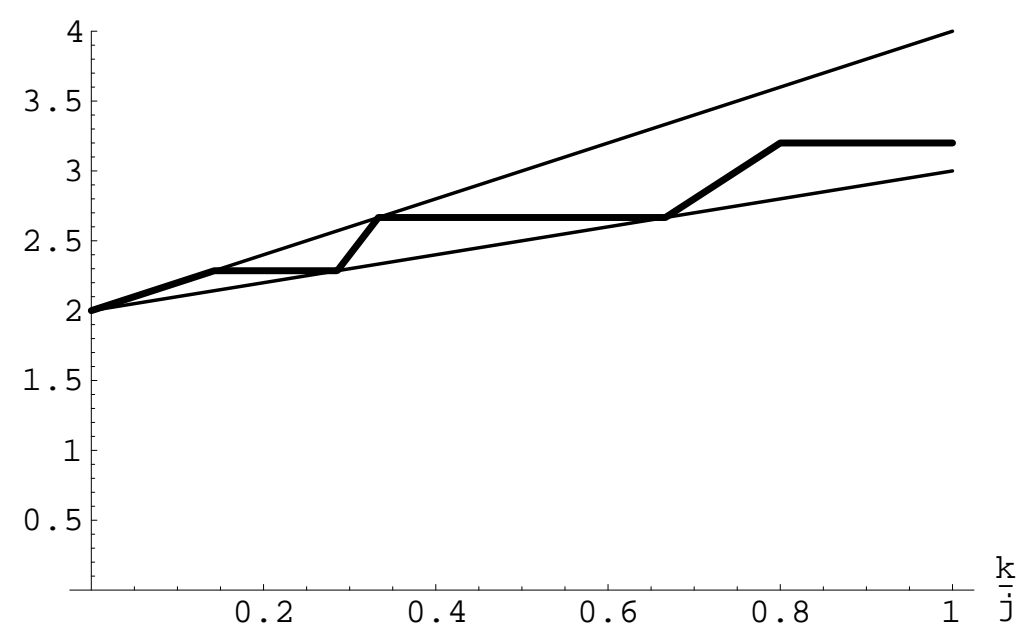


Let $\hat{x}_{i}$ be the remainder when $i j$ is divided by $n j / \alpha$. Thus the sequence $\hat{x}_{i}$ is an "arithmetic progression modulo $n j / \alpha "$ starting at 0 and moving in steps of $j$. Now let

$$
x_{i}=\left\lfloor\hat{x}_{i}\right\rfloor .
$$

Let $f$ be a labelling assigning label $x_{i}$ to vertex $v_{i}$.

Lemma 1 The labelling $f$ satisfies (1) and (2) with span $\lceil n j / \alpha\rceil$.

Proof Consider the sequence $\hat{x}_{i}$ regarded as a relaxed labelling of $C_{n}$ with span $n j / \alpha$. By construction this satisfies (1) and since the upper bound on $\alpha$ ensures that this labelling has span at least $2 j+k,(2)$ is also satisfied. As in the proof of Proposition 1 we can now claim that $f$ is a labelling of $C_{n}$ satisfying constraints (1)-(2) having span $\lceil n j / \alpha\rceil$.

The second type of colouring is called a two-step labelling. Let $\alpha$ be an integer such that

$$
\frac{n j}{2 j+k} \leq \alpha \leq \frac{n-1}{2}
$$

Let $\hat{x}_{i}$ be the remainder when $i k \alpha /(n-2 \alpha)$ is divided by $n k /(n-2 \alpha)$. The sequence $\hat{x}_{i}$ is an "arithmetic progression modulo $n k /(n-2 \alpha)$ " starting at 0 and moving in steps of $\alpha k /(n-2 \alpha)$. As before we now let

$$
x_{i}=\left\lfloor\hat{x}_{i}\right\rfloor
$$

Let $f$ be a labelling assigning label $x_{i}$ to vertex $v_{i}$.

Lemma 2 The labelling $f$ satisfies (1) and (2) with span $\lceil n k /(n-2 \alpha)\rceil$.

Proof Again consider the sequence $\hat{x}_{i}$ regarded as a relaxed labelling $\hat{f}$ of $C_{n}$ with span $\hat{\sigma}=n k /(n-2 \alpha)$. If $u$ and $v$ are adjacent then

$$
|\hat{f}(u)-\hat{f}(v)|_{\hat{\sigma}}=\frac{k \alpha}{n-2 \alpha}
$$

Since

$$
\alpha \geq \frac{n j}{2 j+k}
$$

we see that $\hat{f}$ satisfies constraint (1). Now suppose that $u$ and $v$ are distance two apart. Then

$$
|\hat{f}(u)-\hat{f}(v)|_{\hat{\sigma}}=\hat{\sigma}-\frac{2 k \alpha}{n-2 \alpha}=k .
$$

Hence $\hat{f}$ satisfies constraint (2). Again as in the proof of Proposition 1 we can show that $f$ is a labelling of $C_{2 r}$ satisfying constraints (1)-(2) having span $\lceil n k /(n-2 \alpha)\rceil$. 
We now move on to show that whenever the optimal span is strictly less than $2 j+2 k$, one of these two types of labelling gives the optimal span. Notice that in order for the span to be strictly less than $2 j+2 k$ we need $k>0$. Suppose that $\hat{f}$ is any relaxed labelling of $C_{n}$ satisfying (1) and (2) with span $\hat{\sigma}$ strictly less than $2 j+2 k$. Let $\hat{x}_{i}=\hat{f}\left(v_{i}\right)$ for each $i$. To avoid cumbersome extra cases in the proofs below it is useful to let $\hat{x}_{n}=\hat{x}_{0}$ and $\hat{x}_{n+1}=\hat{x}_{1}$. We may assume without loss of generality that $\hat{x}_{0}=0$ and that $j \leq \hat{x}_{1}<j+k$ for otherwise we can replace $\hat{f}\left(v_{i}\right)$ by $\hat{\sigma}-\hat{f}\left(v_{i}\right)$. Now for $i=0, \ldots, n-1$ define

$$
z_{i}=\left\{\begin{array}{cc}
\hat{x}_{i+1}-\hat{x}_{i} & \text { if } \hat{x}_{i+1}-\hat{x}_{i} \geq 0 \\
\hat{\sigma}+\hat{x}_{i+1}-\hat{x}_{i} & \text { if } \hat{x}_{i+1}-\hat{x}_{i}<0
\end{array} .\right.
$$

Again it is useful to define $z_{n}=z_{0}$. Note that $0 \leq z_{i}<\hat{\sigma}$.

Now let

$$
\alpha=1+\left|\left\{i: 1 \leq i \leq n-1, \hat{x}_{i}<\hat{x}_{i-1}\right\}\right| .
$$

The following lemma contains some results that are useful for finding the lower bound on the span.

\section{Lemma 3}

1 .

$$
\sum_{i=0}^{n-1} z_{i}=\alpha \hat{\sigma}
$$

2. For $i=0,1, \ldots, n-1$,

$$
j \leq z_{i} \leq \hat{\sigma}-j
$$

3. If $k>0$ then for $i=0,1, \ldots, n-1$,

$$
\begin{aligned}
z_{i} & <j+k, \\
z_{i}+z_{i+1}+k & \leq \hat{\sigma} .
\end{aligned}
$$

4. If $k>0$ then $1 \leq \alpha<\frac{n}{2}$.

\section{Proof}

1. This is straightforward using the definitions of $z_{i}$ and $\hat{\sigma}$.

2. Without loss of generality we may assume that $x_{i}=0$. Then in order to satisfy (1) we require $j \leq x_{i+1} \leq \hat{\sigma}-j$. Thus $j \leq z_{i} \leq \hat{\sigma}-j$.

3. We will begin by proving (8) by induction on $i$. We know that $z_{0}<j+k$ by assumption. Suppose we know that $z_{i}<j+k$. We may assume without loss of generality that $x_{i}=0$. Now using the second part of the lemma we see that

$$
x_{i+1}+z_{i+1}=x_{i}+z_{i}+z_{i+1}<j+k+\hat{\sigma}-j=\hat{\sigma}+k .
$$


But in order to satisfy $(2) x_{i+2}$ cannot lie in the interval $[0, k)$ nor in the interval $(\hat{\sigma}-k, \hat{\sigma})$ and so

$$
x_{i+1}+z_{i+1} \leq \hat{\sigma}-k .
$$

This implies that

$$
z_{i+1} \leq \hat{\sigma}-k-x_{i+1}<2 j+2 k-k-j=j+k
$$

as required. Since $x_{i}=0,(10)$ also implies that

$$
z_{i}+z_{i+1}+k \leq \hat{\sigma}
$$

which is (9).

4. By definition $\alpha \geq 1$. Summing (9) over $i$ gives

$$
2 \sum_{i=0}^{n-1} z_{i}+n k \leq n \hat{\sigma}
$$

and so

$$
2 \alpha \hat{\sigma} \leq n(\hat{\sigma}-k)<n \hat{\sigma},
$$

which implies that $\alpha<n / 2$.

We can now prove the key proposition which ensures that whenever the optimal span is strictly less than $2 j+2 k$ then a one-step or two-step labelling is optimal.

Proposition 4 If the labelling $f$ satisfies constraints (1) and (2) with $\alpha=\alpha_{0}$ and span $\sigma$ strictly less than $2 j+2 k$ then

$$
\sigma \geq\left\lceil\max \left\{\frac{n j}{\alpha_{0}}, \frac{n k}{n-2 \alpha_{0}}\right\}\right\rceil .
$$

Proof Let $\hat{f}$ be a relaxed labelling having span $\hat{\sigma}$ strictly less than $2 j+2 k$. We may assume that $\hat{f}\left(v_{0}\right)=0$ and $j \leq \hat{f}\left(v_{1}\right)<j+k$. Thus we can apply Lemma 3 . By the first and second parts of Lemma 3 we see that

$$
\hat{\sigma}=\frac{\sum_{i=0}^{n-1} z_{i}}{\alpha_{0}} \geq \frac{n j}{\alpha_{0}} .
$$

Summing (8) over all $i$ gives $2 \alpha_{0} \hat{\sigma} \leq n(\hat{\sigma}-k)$, just as in the proof of the fourth part of Lemma 3, which implies that

$$
\hat{\sigma} \geq \frac{n k}{n-2 \alpha_{0}} .
$$

Since the labelling $f$ takes only integral values, we see that its span is at least

$$
\left\lceil\max \left\{\frac{n j}{\alpha_{0}}, \frac{n k}{n-2 \alpha_{0}}\right\}\right\rceil .
$$


Proof of Theorem 2 To show that the value in the theorem is an upper bound on the span it is enough to observe that a combination of 1-step labellings, 2-step labellings and labellings with span $2 j+2 k$ satisfy the value in the theorem. Proposition 4 ensures that one of these labellings is optimal. Careful checking of the various possibilities for different values of $\alpha$ is now enough to ensure that the value given in the theorem is indeed optimal.

\section{Odd Cycles}

Calculating the span for odd cycles $C_{2 r+1}$ is more complicated than for even cycles but many of the results obtained for even cycles can be used. The added complication arises because $2 j+2 k$ is no longer an upper bound for $\sigma$, in the same way that an odd cycle is not 2-colourable. We begin by giving the span for odd cycles with $r \geq 4$.

Theorem 3 Let $r$ be an integer such that $r \geq 4$ and, $j$ and $k$ be integers with $0 \leq k \leq j$ then

$$
\sigma\left(C_{2 r+1} ; j, k\right)= \begin{cases}\left\lceil\frac{(2 r+1) j}{r}\right\rceil & 0 \leq \frac{k}{j} \leq \frac{1}{r} \\ (2 r+1) k & \frac{1}{r}<\frac{k}{j} \leq \frac{2}{2 r-1} \\ 2 j+2 k & \frac{2}{2 r-1}<\frac{k}{j} \leq \frac{3}{2 r-2} \\ \left\lceil\frac{(2 r+1) j}{r-1}\right\rceil & \frac{3}{2 r-2}<\frac{k}{j} \leq \frac{3}{r-1} \\ \left\lceil\frac{(2 r+1) k}{2 r+1-2 \alpha}\right\rceil & \frac{2 r+1-2 \alpha}{\alpha}<\frac{k}{j} \leq \frac{2 r+1-2 \alpha}{\alpha-1} \\ \left\lceil\frac{(2 r+1) j}{\alpha}\right\rceil & \frac{2 r-1-2 \alpha}{\alpha}<\frac{k}{j} \leq \frac{2 r+1-2 \alpha}{\alpha} \\ & \alpha=r-2, r-3, \ldots,\left\lceil\frac{2}{3} r\right\rceil .\end{cases}
$$

The graphs of $\hat{\sigma} / j$ as a function of $k / j$ are shown in Figures 4, 5. The lines $y=2 x+1$ and $y=2 x+2$ corresponding to span $2 j+k$ and $2 j+2 k$ are again shown.

Proof Apart from the region where the span is $2 j+2 k$, the span can be achieved by one or two-step labellings. To demonstrate that the value of the span given by the theorem is an upper bound we need to show that when $\frac{2}{2 r-1} \leq \frac{k}{j} \leq \frac{3}{2 r-2}$ it is possible to label $C_{2 r+1}$ with span $2 j+2 k$. We briefly sketch how such a labelling is constructed. Using the notation of Section 3, we construct a labelling with span $2 j+2 k$ by setting $z_{i}=j+\delta_{i}$, where the $\delta_{i}$ are still to be chosen and satisfy $0 \leq \delta_{i} \leq 2 k$. To ensure that the labelling has span $2 j+2 k$ we require $\sum_{i} \delta_{i}=m$ where $m=(-(2 r+1) j) \bmod (2 j+2 k)$. 
Figure 4: Graph of $\hat{\sigma} / j$ against $k / j$ for $C_{9}$

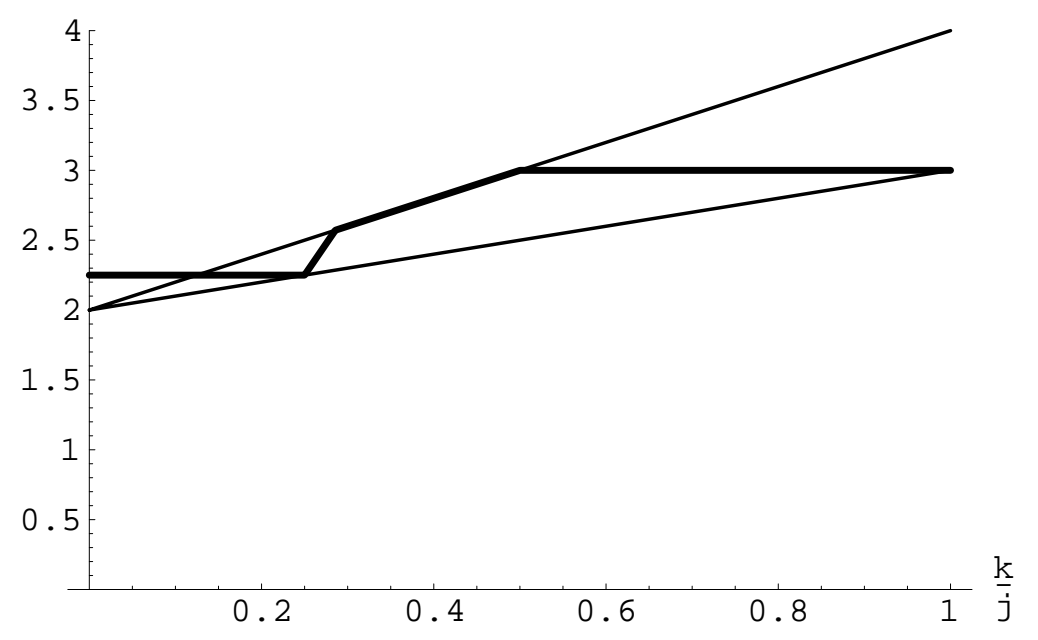

Figure 5: Graph of $\hat{\sigma} / j$ against $k / j$ for $C_{17}$

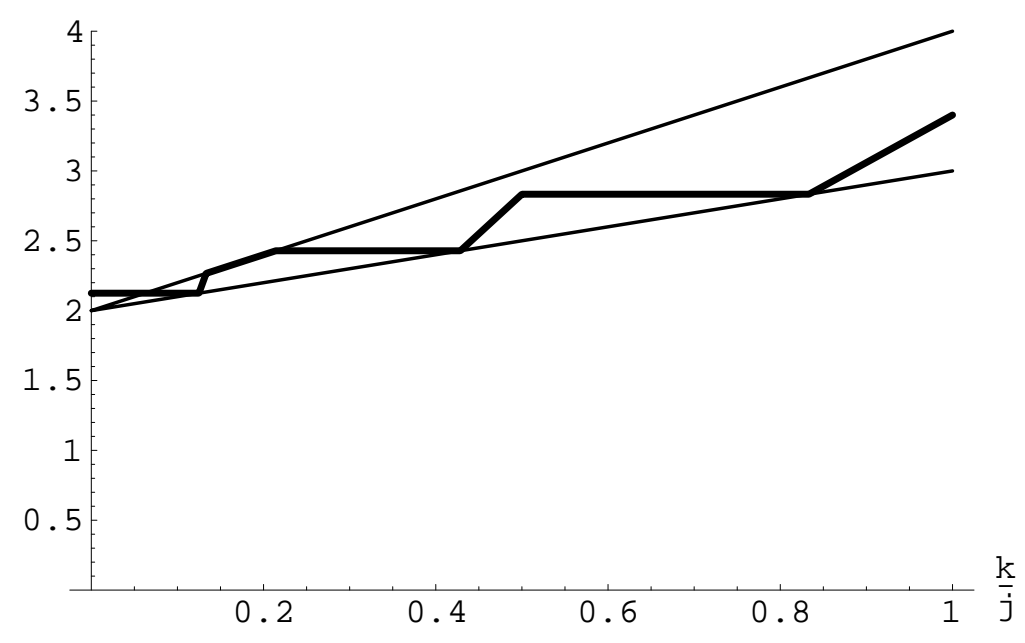


In the region where

$$
\frac{2}{2 r-1} \leq \frac{k}{j} \leq \frac{3}{2 r-2},
$$

$2 j+2 k \leq(2 r+1) k$ and so $1 \leq m \leq(2 r+1) k-1$. If $m$ is of the form $2 s k+t$ for positive integers $s$ and $t$ with $0 \leq t<k$, then a possible choice of the $\delta_{i}$ satisfying (1)and (2) is given by

$$
\delta_{1}=\delta_{s+1}=k, \delta_{2}=\ldots=\delta_{s}=2 k, \delta_{s+2}=0, \delta_{s+3}=t, \delta_{0}=\delta_{s+4}=\ldots=\delta_{2 r}=0 .
$$

Since $s \leq r$ and $r \geq 4$ this is always possible, that is the cycle is large enough to allow us to do this. If $m$ is of the form $(2 s+1) k+t$ for positive integers $s$ and $t$ with $0 \leq t<k$, then a possible choice of the $\delta_{i}$ satisfying (1) and (2) is given by

$$
\begin{gathered}
\delta_{1}=\delta_{s+1}=\delta_{s+3}=k, \delta_{2}=\ldots=\delta_{s}=2 k, \\
\delta_{s+5}=t, \delta_{0}=\delta_{s+2}=\delta_{s+4}=\delta_{s+6}=\ldots=\delta_{2 r}=0 .
\end{gathered}
$$

Since $s \leq r-1$ and $r \geq 4$ this is always possible, that is the cycle is large enough to allow us to do this.

Apart from the first interval, the span given in the theorem is at most $2 j+2 k$. Careful checking shows that the span is always the minimum that can be achieved by a onestep labelling, a two-step labelling or $2 j+2 k$. Hence Proposition 4 implies that the theorem gives the optimal span except possibly for part of the first interval. The proof of Proposition 4 implies that when $k / j=\frac{1}{r}$,

$$
\hat{\sigma}\left(C_{2 r+1} ; j, k\right)=\frac{(2 r+1) j}{r} .
$$

Now consider the value of $\hat{\sigma}\left(C_{2 r+1} ; j, 0\right) / j$. This is the circular chromatic number studied in [6] and is equal to $(2 r+1) / r$. Hence

$$
\hat{\sigma}\left(C_{2 r+1} ; j, 0\right)=\frac{(2 r+1) j}{r},
$$

and since, for a fixed value of $j, \hat{\sigma}$ is an increasing function of $k$, we deduce that if $k / j \leq 1 / r$ then $\hat{\sigma}\left(C_{2 r+1} ; j, k\right)=(2 r+1) j / r$. This is enough to imply that the theorem gives the correct value in the first interval.

The following theorem completes matters by giving the span for $C_{3}, C_{5}$ and $C_{7}$.

Theorem 4 Let $j$ and $k$ be integers such that $0 \leq k \leq j$.

$$
\begin{aligned}
& \sigma\left(C_{3} ; j, k\right)=3 j, \\
& \sigma\left(C_{5} ; j, k\right)= \begin{cases}\left\lceil\frac{5 j}{2}\right\rceil & 0 \leq \frac{k}{j} \leq \frac{1}{2} \\
5 k & \frac{1}{2}<\frac{k}{j} \leq 1,\end{cases}
\end{aligned}
$$


Figure 6: Graph of $\hat{\sigma} / j$ against $k / j$ for $C_{7}$

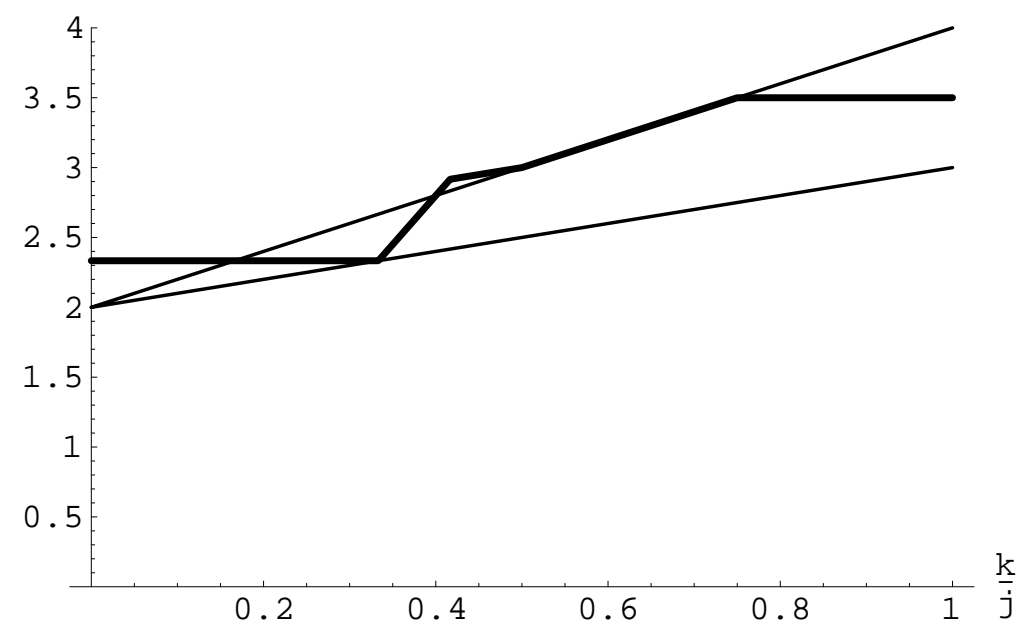

$$
\sigma\left(C_{7} ; j, k\right)= \begin{cases}\left\lceil\frac{7 j}{3}\right\rceil & 0 \leq \frac{k}{j} \leq \frac{1}{3} \\ 7 k & \frac{1}{3}<\frac{k}{j} \leq \frac{5}{12} \\ \left\lceil\frac{5 j}{2}+k\right\rceil & \frac{5}{12}<\frac{k}{j} \leq \frac{1}{2} \\ 2 j+2 k & \frac{1}{2}<\frac{k}{j} \leq \frac{3}{4} \\ \left\lceil\frac{7 j}{2}\right\rceil & \frac{3}{4}<\frac{k}{j} \leq 1\end{cases}
$$

The graph of $\hat{\sigma} / j$ is shown in Figure 6 .

In the part of the proof dealing with $C_{7}$ we will need the following proposition, which is a modification of the Travelling Salesman Bound.

Proposition 5 Let $G$ be a graph with vertex set $\left\{v_{1}, \ldots, v_{n}\right\}$ and let $\pi$ be an ordering of these vertices. For any subset $\left\{\pi\left(i_{1}\right), \ldots, \pi\left(i_{r}\right)\right\}$ of the vertices, where $1 \leq i_{1}<\cdots<i_{r} \leq$ $n$,

$$
\sigma(G, \pi ; j, k) \geq \sum_{j=1}^{r-1} c_{\pi\left(i_{j}\right), \pi\left(i_{j+1}\right)}+c_{\pi\left(i_{r}\right), \pi\left(i_{1}\right)} .
$$

Proof of Theorem 4 To show that $\sigma\left(C_{3} ; j, k\right)=3 j$ we observe that labelling the vertices with $0, j$ and $2 j$ gives a labelling with span $3 j$. Proposition 2 shows that $3 j$ is a lower bound.

For $C_{5}$ we use Proposition 2 to show that $5 k$ is a lower bound for the span. The circular chromatic number of $C_{5}$ is $5 / 2$ and so $\lceil 5 j / 2\rceil$ is also a lower bound for the span. 
When $k / j \leq 1 / 2$ we can label the vertices in turn, moving around the cycle using labels $0, j, 2 j,\lceil j / 2\rceil,\lceil 3 j / 2\rceil$ giving span $\lceil 5 j / 2\rceil$. When $k / j>1 / 2$ we can label the vertices in turn, moving around the cycle using labels $0,2 k, 4 k, k, 3 k$ giving span $5 k$.

Finding $\sigma$ for $C_{7}$ is the most complicated part of all because the cycle is not large enough to allow us to use the method of Theorem 3 to find a labelling of span $2 j+2 k$. The arguments used in Theorem 3 show that the value of the span given by the theorem is correct except possibly for

$$
\frac{k}{j} \in\left(\frac{2}{5}, \frac{3}{4}\right)
$$

when the span of the best one or two-step labelling exceeds $2 j+2 k$. By modifying the argument in the proof of Theorem 3 we can show that there is a labelling with span $2 j+2 k$ when $k / j \geq 1 / 2$.

To complete the proof we must find the optimal labelling when $2 / 5<k / j<1 / 2$. The existence of a labelling with span $2 j+2 k$ for $k / j \geq 1 / 2$ implies that when $k / j=1 / 2$ there is a labelling with span $3 j$. This labelling will satisfy (1) and (2) whenever $k / j \leq 1 / 2$. Furthermore the two-step labelling that is optimal when $k / j \leq 2 / 5$ remains feasible for larger values of $k / j$.

Thus we need to determine whether there exists a labelling with span less than $\min \{7 k, 3 j\}$. To answer this question, we consider the minimum span $\sigma\left(C_{7}, \pi ; j, k\right)$ with ordering $\pi$, as introduced in the proof of Proposition 1. We first observe that if the span is to be less than $7 k$ then $\pi$ must contain at least one 'long' edge, meaning that $d_{C_{7}}(\pi(i), \pi(i+1))$ is equal to three for some $i$.

Proposition 5 effectively takes a set of edge-disjoint paths from the cycle $(\pi(1), \ldots, \pi(n))$ that corresponds to $\pi$, and calculates a bound based on the proximity of their end-vertices. Among all orderings containing a long edge (and removing obvious symmetries) there are only two that do not contain three edge-disjoint paths with adjacent end-vertices, and which are therefore the remaining candidates for a span of less than $3 j$. They are $\pi_{1}=\left(v_{1}, v_{4}, v_{6}, v_{2}, v_{7}, v_{3}, v_{5}\right)$ and $\pi_{2}=\left(v_{1}, v_{3}, v_{5}, v_{2}, v_{7}, v_{4}, v_{6}\right)$, where the $i$ th component in each 7 -tuple is the vertex $\pi(i)$. For $\pi_{2}$, using the subset $\left\{v_{1}, v_{3}, v_{2}, v_{7}, v_{6}\right\}$ in Proposition 5 establishes a bound of $2 j+3 k$, which is at least $3 j$ for $k / j \geq 1 / 3$. For ordering $\pi_{1}$, the minimum span may be found using an algorithm of McDiarmid [3], which for $k / j \leq 1 / 2$ gives

$$
\sigma\left(C_{7}, \pi_{1} ; j, k\right)=\left\lceil\frac{5}{2} j+k\right\rceil,
$$

corresponding to the assignment

$$
\left(f\left(v_{i}\right): i=1, \ldots, 7\right)=\left(0, j, 2 j,\left\lfloor\frac{1}{2} j\right\rfloor, 2 j+k,\left\lfloor\frac{1}{2} j+k\right\rfloor,\left\lfloor\frac{3}{2} j+k\right\rfloor\right) .
$$

This is an improvement over a span of $7 k$ for $\frac{k}{j} \in\left(\frac{5}{12}, \frac{1}{2}\right)$, with $7 k$ remaining the best for $\frac{k}{j} \in\left(\frac{2}{5}, \frac{5}{12}\right)$. 


\section{Conclusions}

The results in this paper give a complete specification of the minimum span of a labelling of a cycle with constraints at two distances. Although the value of the span is complicated, most of the labellings used to achieve it can be specified very easily. The complexity of the span suggests that it would be difficult to obtain similar results for other classes of graphs.

One of the original motivations of this paper was to investigate the span of subgraphs of the square lattice with constraints at two distances. The even cycles form a subclass of these graphs. Our results completely solve the case of subgraphs of the square lattice with maximum degree at most 2 . In the case when the maximum degree is 4 the optimal span is $2 j+3 k$. The travelling salesman bound (Proposition 2) shows that this is a lower bound and it is shown in [2] that the span of the infinite square lattice is $2 j+3 k$. It is not possible to obtain any general results when the maximum degree is 3 because a theorem independently obtained by Gräf [1] and Shepherd [5] implies that finding the optimal span of a subgraph of the square lattice with $j=k=1$ is NP-hard.

\section{References}

[1] A. Gräf. Colouring and recognising special graph classes. PhD thesis, University of Mainz, 1994.

[2] J. van den Heuvel, R. A. Leese, and M. A. Shepherd. Graph labelling and radio channel assignment. Journal of Graph Theory, 29:263-284, 1998.

[3] C. J. H. McDiarmid. A doubly cyclic channel assignment problem. Discrete Applied Mathematics, 80:263-268, 1997.

[4] A. Raychaudhuri. Intersection assignments, T-colourings and powers of graphs. $\mathrm{PhD}$ thesis, Rutgers University, 1985.

[5] M. A. Shepherd. Radio Channel Assignment. DPhil thesis, University of Oxford, 1996.

[6] A. Vince. Star chromatic number. Journal of Graph Theory, 12:551-559, 1988. 